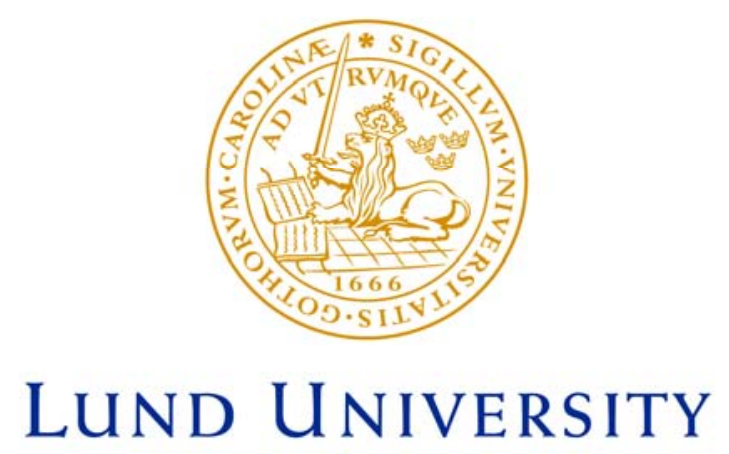

Faculty of Medicine

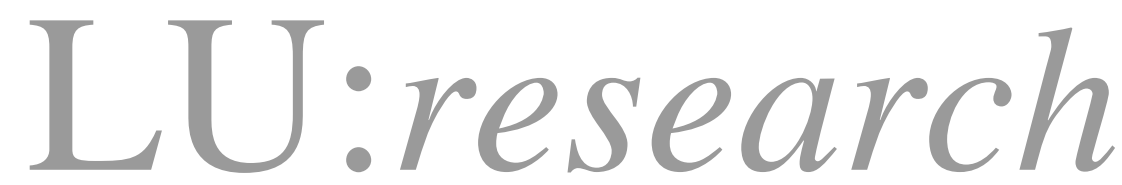

Institutional Repository of Lund University

This is an author produced version of a paper published in Journal of health psychology. This paper has been peerreviewed but does not include the final publisher proofcorrections or journal pagination.

Citation for the published paper:

Ekelund, Marie-Louise and Andersson, Sven Ingmar. "Elucidating issues stressful for patients in predialysis and dialysis: from symptom to context" Journal of health psychology, 2007, Vol: 12, Issue: 1, pp. 115-26.

Access to the published version may require journal subscription.

Published with permission from: Sage Publications 


\section{Elucidating Issues Stressful for Patients in Predialysis and}

\section{Dialysis: From Symptom to Context}

\section{MARIE-LOUISE EKELUND}

Department of Nephrology, Clinical Sciences, Lund University, Lund, Sweden.

\section{SVEN INGMAR ANDERSSON}

Department of Psychology, Lund University, Lund, Sweden.

MARIE-LOUISE EKELUND is a graduate student at the Department of Nephrology, Clinical Sciences, Lund University, Lund, Sweden.

SVEN INGMAR ANDERSSON is Professor of Psychology at Lund University, Lund, Sweden.

ACKNOWLEDGEMENTS. The study was supported by grants from The Bank of Sweden Tercentenary Foundation, The Vardal Foundation, The Swedish Research Council, and from the health care authorities in Region Skåne in Sweden.

COMPETING INTERESTS: None declared.

ADDRESS. Correspondence should be directed to: and offprint request to:

MARIE-LOUISE EKELUND, Department of Nephrology, Clinical Sciences, Lund University, SE-221 85 Lund, Sweden. [Tel: +46 46 171734; email: Marie-Louise.Ekelund@med.lu.se]

Running head: Stressful issues in predialysis and dialysis patients 


\begin{abstract}
Biopsychosocial problems experienced by renal disease patients were studied within a contextual framework the patients themselves defined, the findings being related to sociodemographic and medical data. Participants were 72 predialysis patients and 73 patients being treated by haemodialysis or peritoneal dialysis (106 men and 39 women, aged 18-84 years). Both stress-related global and situational measures of biopsychosocial problems were assessed by questionnaire. Factor analyses revealed five factors - Bodily problems; Work and leisure time; Sleep, cramps and itching complaints; Financial problems; and Dependence - explaining $71.1 \%$ of the total variance. Fatigue loaded both on Bodily problems and Work and leisure time. Sleep disturbances, itching of the skin and muscle cramps were more stressful for the dialysis than the predialysis patients. Also, fatigue, inability to work, and limitation on vacation activities appeared more stressful for the dialysis group. The study supports the usefulness of a contextual approach for gaining a better general understanding of renal disease patients' problems.
\end{abstract}

\title{
Keywords
}

biopsychosocial variables, contextual approach, dialysis, nephrological care, patient self-assessment, predialysis 


\section{Introduction}

IN PATIENTS with renal disease, psychological and psychosocial well-being may contribute importantly if treatment is to be successful (e.g. Devins, Mann, Mandin, Paul, Hons, Burgess, Taub, Schorr, Letourneau, \& Buckel, 1990; Unruh, Weisbord, \& Kimmel, 2005). Maintenance of the quality-of-life of patients is also seen as a primary goal in renal medicine (McGee \& Bradley, 1994). Although the use of quality-of-life measures has increased considerably in recent decades, the mean values for such measures regarded as satisfactory have not been agreed upon for patients with renal disease (e.g. Edgell, Coons, Carter, Kallich, Mapes, Damush, \& Hays, 1996; Kimmel, 2000a; Sensky, 1993). As Kimmel (2000b) points out, there is a need not only of understanding demographic and medical factors in these patients and how these relate to outcomes, including adjustment, compliance, morbidity and mortality, but also of understanding the psychosocial factors involved, which may also represent critical elements in the quality of their treatment and in their survival. Such a broad elucidation of different factors, which is generally lacking, can be regarded as representing a key to the success of individual and multidisciplinary rehabilitation programmes (Andersson, 1996).

In Sweden, where the study was undertaken, the yearly incidence of patients receiving renal replacement therapy has been relatively stable, about 125 per 1,000,000 inhabitants receiving it, ever since 1990, when statistics first became available (SRAU, 2002). From these statistics it is also evident that the numbers of such patients older than 65 years of age and of patients with diabetes mellitus type II have tended to increase. 
The present study aims at elucidating the medical, psychological and psychosocial factors experienced as particularly stressful by renal disease patients and to evaluate the impact of such factors on predialytic and on dialytic patients, respectively.

\section{Subjects and methods}

\section{Study Population}

The study population consisted of 201 renal disease patients in southern Sweden, aged $18-84$ years, who took part in a renal education and rehabilitation programme earlier, 145 (72\%; 106 men and 39 woman) participating in the study.

\section{Procedure}

All participants were provided written and oral information regarding the study and were also informed that participation was voluntary, that confidentiality was assured and that they could withdraw at any time without this interfering with their treatment or care. Participants were given a questionnaire developed for studying stress, appraisal and coping. They also completed a questionnaire concerning routine demographic information. Diagnostic information concerning the patients was obtained from their medical records.

\section{Questionnaires}

Two questionnaires were given:

1. A questionnaire for obtaining routine demographic information of the type shown in Table 1.

2. A questionnaire developed by the second author (SIA) for studying the frequency and severity of stressors reported in the literature to be prevalent in various groups of chronic patients and for examining situational patterns of appraising stressful episodes and coping with them (Andersson \& Albertsson, 
2000; Andersson, Manthorpe, \& Theander, 2001). The questionnaire, referred to here as the "instrument," included a list of 87 factors representing either predominantly biological/somatic stressors (e.g., muscle cramps, itching of the skin, muscle weakness, or fatigue) or predominantly psychological/ psychosocial stressors (e.g. uncertainty regarding the future, fatigue, or effects on sexuality). Subjects were to indicate for each of the stressors whether it had occurred during the past week (yes or no) and the extent to which it had been experienced as stressful (on a 0 to 100 scale on which $0=$ not stressful at all and $100=$ extremely stressful). Subjects were also given the possibility of listing additional stressors they considered to be lacking in the original set and evaluating them. The instrument involved each subject's selecting what he/she considered to be the three most stressful factors and to describe for each a typical situation in which this factor (stressor) was involved. For each of these three types of situations the subject was to describe what he/she tended to feel (emotional appraisal), think (cognitive appraisal), attempt to do (intention) and to actually do (coping) under such circumstances.

\section{Statistical analysis}

Data analysis involved obtaining frequencies (\%), means and standard deviations, and conducting a factor analysis, as well as performing chi-square tests and analysis of variance for studying differences between those who were in predialysis and those who had started dialysis. For comorbidity, a simple "yes" or "no" measure was employed with regard to the presence of other chronic disease (e.g., cancer, cardiovascular disease, chronic pulmonary disease, diabetes mellitus, rheumatoid arthritis), based on inspection of the patients' medical records. In the factor analyses, factor extraction involved principal component analysis on the basis of the eigenvalue-greater-than-one criterion, subsequent varimax rotation being used for the orthogonal transformation of the factors. 


\section{Results}

\section{Demographic and clinical characteristics}

Of the total of 201 patients included in the study, 145 patients (72.2\%) participated, 72 predialytic and the remaining 73 patients being treated with haemodialysis $(N=57)$ or peritoneal dialysis $(N=16)$. Table 1 shows the age and sex, and the marital, socioeconomic and clinical status (e.g., diabetic or non-diabetic) of the patients who participated. Non-diabetic patients were categorized as predialytic if their serum creatinine concentration was $>300-350 \mu \mathrm{mol} / 1$, the corresponding level for inclusion of diabetic patients as predialytic being $>250 \mu \mathrm{mol} / 1$. The dialysis patients were employed to a somewhat lesser extent than the predialysis patients $(P<0.05)$. Also, the mean duration of kidney disease in the predialysis sample of women was longer than the corresponding mean for the women in dialysis $(P<0.05)$.

\section{Stressful problems: factor analysis}

Of the types of problems that were indicated as being experienced, 23 were experienced by at least $20 \%$ of the total patient group. Five types of problems originally included in a preliminary factor analysis and found to load on three or more of the factors were discarded from further analysis. These were aching, pain, shortness of breath, being away from their family, and numbers of visits to the hospital or stays there, the mean value for each being between 31.0 and 34.4. The stressfulness data (0-100 scores) for the remaining 18 items were subjected to factor extraction through principal component analysis involving use of an eigenvalue-greater-than-one criterion. In the factor extraction, five factors that together explained $71.1 \%$ of the total variance were retained, being labelled as follows: Factor 1: Bodily problems (43.7\%); Factor 2: Work and leisure time (8.7\%); Factor 3: Sleep, cramps and itching complaints (7.6\%); Factor 4: Financial problems (5.9\%); and Factor 5: Dependence (5.2\%). Table 2 shows the outcome of the varimax rotation of the five factors and the significant differences obtained between the predialysis 
and dialysis patient groups on individual items. Cronbach alphas were computed for each factor sub-scale comprising items with factor loadings greater than 0.377 . For Factors $1-5$, the alpha values were 0.89 , $0.81,0.77,0.85$, and 0.76 , respectively.

Stressful problems: predialysis vs. dialysis

Frequency of problems As is evident in Table 2, the problems were generally more frequently endorsed by the dialysis than the predialysis group, significantly so $(P<0.05)$ so for 10 of these, with sleep disturbances, effects on sexuality, and inability to work all being represented in more than $60 \%$ of the dialysis patients. Fatigue was the type of problem most frequently reported, by more than $70 \%$ of the subjects in both groups, there being no significant difference between the groups on this measure.

Stressfulness score Means were generally greater for the dialysis than the predialysis group, significantly so $(\mathrm{p}<0.05)$ for sleep disturbances, itching of the skin, inability to work, fatigue, limitation on vacation activities, and muscle cramps. In a descriptive sense it was noted that the highest mean stressfulness score obtained in the dialysis patient group was for inability to work and effects on sexuality, both $>50.0$, and that in the predialysis group only one stressor, effects on sexuality, had a mean score $>40.0$. Only for one of the types of problems originally included in the factor analysis but later dismissed, being away from the family, was a significant difference $(P<0.05)$ obtained between the predialysis and the dialysis patient groups, mean $(\mathrm{SD})=29.4(30.2)$ and $34.9(32.7)$, respectively.

\section{Situational accounts}

Altogether 56 of the patients, 23 of them in predialysis (15 men, 8 women) and 33 in dialysis ( 23 men, 10 women), provided 158 situational accounts. 
Factor 1: Bodily problems Most of the 11 accounts pertaining to this type of problem were provided by patients undergoing dialysis. Five of the accounts, related to muscle weakness, having limited control over bodily function or limitation of movement, were given by patients in dialysis who had a comorbidity of diabetes mellitus.

Example: D51. Man, aged 50.5. Education: 7 years. Unmarried. Retired, formerly self-employed. In dialysis for 3 years.

Stress factor: Limited control over bodily function.

Situation: When I'm out walking, my legs can suddenly give way under me so that I fall down headfirst, without any forewarning.

Feeling: Hopeless.

Thinking: It's hell, but I don't want to give up.

Trying to do: Considering how to get up again and go on.

Doing: I have to take care of things myself.

Help: People think I'm intoxicated.

Assessment of help: People don't help me.

Accounts of both by the predialytic and the dialytic patients pertaining to fatigue generally concerned decline in bodily function, such as being tired even after exerting little effort, or being in poor physical shape.

Example: PD46. Man, aged 57.5. Education: 6 years. Married. Retired, formerly an unskilled worker. In predialysis.

Stress factor: Fatigue. 
Situation: When having mowed the lawn.

Feeling: That my physical condition is so poor.

Thinking: I want to sit down.

Trying to do: Rest.

Doing: Sitting down.

Help: I take medicine for my anemia.

Assessment of help: Good.

Example D04. Woman, aged 19.5. Education: 12 years. Unmarried. Student. In dialysis for 3 years.

Stress factor: Fatigue.

Situation: In school, after receiving dialysis.

Feeling: I feel more tired than I usually do-although I always feel tired.

Thinking: Nothing works! I keep trying out different headache tablets.

Trying to do: Rest.

Doing: Resting, taking headache tablets.

Help: None.

Assessment of help: Negative.

Factor 2: Work and leisure time All six of the accounts relating to work were given by patients in dialysis. With regard to limitations experienced during vacation activities, an additional two accounts concerned uncertainty related to future planning.

Example D58 Man, aged 62. Education: 9 years. Married. Retired, an unskilled worker. In dialysis for 1 year. 
Stress factor: Limited work capacities.

Situation: Having no work to go to.

Feeling: Being an outsider.

Thinking: You have to find something else to do.

Trying to do: Going on as usual.

Doing: Working in the garden.

Help: If you find some kind of hobby, things work out.

Assessment of help: There are so many different hobbies and sports activities I can take part in.

As group means in Table 2 indicate, effects on sexuality was a highly stressful factor for patients both in predialysis and in dialysis. The accounts relating to this factor reflect the considerable stress this involved. In all cases, such accounts were only given by men, who made such statements as, "I don't feel able to do very much as a man in such a situation". Other patients described their feelings of insufficiency in sexual situations.

Example PD36 Man, aged 50.5. Education: 8 years. Married. On sick-leave, an unskilled worker. In predialysis.

Stress factor: Effects on sexuality.

Situation: I want to have sex. My body doesn't want to.

Feeling: Sad. I wonder what will happen.

Thinking: I hope it will be solved.

Trying to do: Accept it.

Doing: Trying to sleep. We talk about it a little.

Help: Being considerate about it, perhaps. 
Assessment of help: Don't know if it helps.

Example D40. Man, aged 62.4. Education: 8 years. Married. Retired, unskilled worker. In dialysis for 4 years.

Stress factor: Effects on sexuality.

It's difficult for it to not work out. I speak with my wife about it. I've gotten some help from a urologist, something that I inject. I don't know if it still works. Nobody has discussed it with me. It would be good if somebody did.

Factor 3: Sleep, cramps and itching complaints The factor analysis showed factors relating to muscle cramps and itching of the skin to be closely associated with those of sleep disturbances and the effects of their illness on family life. Various problems of this kind were described, by the dialysis patients in particular. Fatigue was often attributed to itching or muscle cramps.

Example D09. Man, aged 70.0. Education: 7 years. Married. Retired, manual worker. In dialysis for 10 years.

Stress factor: Sleep disturbances.

Situation: Can't sleep because of itching. I wake up 3 or 4 times a night.

Feeling: Being powerless.

Thinking: If only it would stop soon.

Trying to do: Get to sleep again.

Doing: Sooner or later I fall asleep.

Help: None.

Assessment of help: Negative. 
Problems of itching were described variously as appearing during dialysis, as only appearing occasionally and then anywhere on the body, or as appearing in the evening at bedtime only. One patient reported that he had found out himself that his itching must be related to his kidney problems, adding "but nobody has explained this for me". The skin creams or ointments given to the patients were often considered to be of little or no help for their itching. Although the stress of the effects their illness had on their family life was one of the items loading on this factor, it was noted that the patients made no direct reference in their situational descriptions to the effects of their problems of sleeping, cramps or itching on family life, even if efforts they made at night to improve their situation were often referred to, such as "up walking around", “massage”, "reading”, or "playing patience."

Example D23 Woman, aged 45 years. Education: 9 years. Separated, with two daughters still living at home. Retired, an unskilled worker. In dialysis for 12 years.

Stress factor: Sleep disturbances.

Situation: Not being able to sleep until early in the morning.

Feeling: Anguished.

Thinking: Of everything and everybody.

Trying to do: Playing patience, reading, shutting my eyes.

Doing: Walking around.

Help: Sleeping tablets.

Assessment of help: The tablets don't help.

Factor 4: Financial problems Most of the examples given here concerned income reduction or economic costs due to the illness. Examples: (D53, woman) "The income benefits now are higher than they were when I got ill. Why should this happen to me?"; (D125, man) "I get paid less now than before 
when I'm ill. I feel powerless. If I was healthy, there wouldn't be any drop in my income.” Uncertainty regarding the future often concerned problems involved in waiting for transplantation.

Factor 5: Dependence This factor related both to being dependent upon one's spouse and one's children, as evident already in the predialytic patients ("feeling dependent increases a lot when I'm in acute need of starting dialytic treatment"), and to having to visit the hospital and meet with the physicians there. Feelings and thoughts of being weak, dejected, deserted or unsafe were particularly salient. Generally, the behaviour reported by the patients was avoidant or passive, although occasionally the account of being dependent upon others and the uncertainty associated with having to take special medication involved references to physical training as a way of coping.

Additional findings Generally, the feelings expressed by the patients concerned a wide spectrum of feelings involving sadness or fear. Anger, however, was not expressed in any of the situational descriptions that the women in the sample provided, whereas it was found in $20.1 \%$ of the descriptions provided by the males, mainly by those in the dialytic group. Descriptions of anger in the male patients in that group related mainly to problems of itching, weight or memory, or to fistular problems. Problems of sexuality in the male patients resulted in expressions of anger, sadness or fear.

\section{Discussion}

There appear to be many advantages in using the instrument described here for documenting various types of illness-related complaints in patients with renal disease. Generally, the patients had no problems in completing the stressor parts of the instrument (yes/no; 0-100 degree scale). The stressors listed appeared to be comprehensive enough for the sample tested. Also, the sub-scales identified through principal component analysis were found to be satisfactory, as evidenced by none of the Cronbach alphas 
being below 0.76 . One could argue, perhaps, that the sub-scales thus identified could be used for comparing differences between the dialysis and the predialysis patients. However, as some of the items loaded on two factors, such an approach was discarded in favour of analyzing scores on individual items. The part of the instrument requiring subjects to describe situationally-related appraisal and coping appeared somewhat more difficult for the patients to fill in than the part involving lists of stressors only. One could argue that for the present patient group, additional interviews, using the same basic format as in the instrument, would best be carried out so as to elucidate problems more adequately in patients having difficulties in expressing their ideas in writing. In the literature, end-stage renal disease has been associated with a wide range of neurocognitive impairments which, although subtle, can have a significant impact upon everyday functioning. Also, there is a need of elucidating renal disease patients' thoughts, feelings and reactions in order to better understand the relationship between psychosocial, demographic and medical factors in renal disease, which might also be able to contribute to such patients' longevity (Kimmel, 2001; Unruh et al., 2005). The present type of instrument can provide a useful means of putting patients' symptoms and problems in the context of their everyday life and of studying health and illness in a patient-centred way as an aid to optimising the overall outcome of treatment (Sensky, 1993).

The importance of symptoms in kidney disease has been highlighted by Parfrey, Vavasour, Henry, Bullock and Gault (1988), Weisbord, Fried, Arnold, Rotondi, Fine, Levenson and Switzer (2004), and Unruh et al. (2005), for example. Parfrey et al. (1988) listed tiredness, cramps, pruritus, dyspnea, headaches and joint pain as the six most important symptoms in dialysis patients, finding little difference in the prevalence of these symptoms between hemo- and peritoneal dialysis patients. All six symptoms were included in our list. However, in our study, pain and shortness of breath (dyspnea) were eliminated through factor analysis, their being found to load on three or more of the factors. Nevertheless, one could argue that pain and dyspnea should be included in questionnaire checklists for studying symptoms in 
ESRD patients in connection with changes in treatment modalities (see Parfrey et al., 1988)., Weisbord et al. (2004) developed a 30-item assessment index of symptoms in patients with chronic hemodialysis. Although their index included types of problems we also found to be common in ESRD patients, such as "fatigue", "effects on sexuality", and "muscle cramps", they did not take up problems that were found on our questionnaire to be prevalent, such as "limitation on vacation activities", "effects of illness on family life", "economic costs", "uncertainty regarding the future", "being dependent on physicians", and "being dependent upon others". Other items included in our questionnaire, such as "limited control over bodily function", "loss/limitation of bodily function", "limited work capacities", and "inability to work" appeared to be represented in the Weisbord et al. instrument by the symptom "feeling tired or lack of energy" but were nevertheless found in our factor analyses to represent a heterogenous group. On the other hand we did not find "nausea", "vomiting", "diarrhea", "swelling of legs", "shortness of breath", or “dizziness", described by Weisbord et al. as being bothersome symptoms in chronic hemodialysis, to be particularly common in the ESRD patients we studied. This suggests that the Weisbord et al. (2004) assessment index, although developed specifically for patients dependent on hemodialysis, may not be comprehensive enough to provide an understanding of the issues faced by patients with ESRD. Their assessment index, as Weisbord et al. point out, requires additional psychometric testing prior to any widespread use.

In our study, fatigue loaded on the factors of both Bodily problems and Work and leisure time. The descriptive and cross-sectional nature of the study does not allow one to infer the direction of the relationship between fatigue and the various other problems involved, such as being able to conclude that the inability to work causes fatigue or that fatigue affects sexuality. Sexual dysfunction appeared to be one of the most stressful problems for the patients here, particularly for the dialysis patients. This emphasizes the importance of health care workers being open to the topic of sexuality in renal disease patients and their partners (Ekelund, Westman, \& Andersson, 2004). Accounts such as that given by 
Patient D40 illustrate the need of assessing sexual concerns in renal disease patients and of providing sexual information, counselling, and referral (Palmer, 1999). The difficulties which the bodily, work and leisure-time problems involve may have had reciprocal effects on each other and have acted as mediators, contributing to the significantly higher mean stressfulness scores obtained for the dialysis than for the predialysis patients. Identification of the various factors found to be related to fatigue also emphasizes the importance of intervention strategies for dealing with these aspects of care (McCann \& Boore, 2000). The present instrument could provide health care personnel information useful for enhancing the quality of life of their patients.

In a study of patients with end-stage renal disease who were on hemodialysis, Brunier and Graydon (1993) found inactivity to contribute to fatigue more than frequency of symptoms did. They argued that good symptom management can reduce fatigue in hemodialysis patients appreciably. In our study, patients' accounts of ways in which they managed their symptoms often referred to their being inactive, such as "resting" or "sitting down". This emphasizes the value of physical exercise programs for promoting general fitness in renal disease patients. Such training may be particularly important for predialytic patients (Wiberg, 2003).

Sleep disturbances were frequent in both the predialysis and the dialysis patients, the dialysis patients in particular complaining of such problems. As Iliescu, Coo, McMurray, Meers, Quinn, Singer and Hopman (2003) have pointed out, poor sleep is common in dialysis patients and is associated with a lower health-related quality of life. In our study, sleep disturbances occurred in the dialysis patients with a frequency of $67.2 \%$, which is comparable to the $40-80 \%$ prevalence reported in previous studies of such patients (Iliescu et al., 2003; Williams, Tell, Zheng, Shumaker, Rocco, \& Sevick, 2002). The situational descriptions relating to poor sleep that some of the patients provided could be interpreted as matching the criteria for specific sleep disorders (sleep apnea, periodic limb movement disorder, and restless legs syndrome) (Parker, 1997). Itching of the skin, muscle cramps, and the effects of the illness on family life 
loaded on the same factor as sleep disturbances. This emphasizes the importance of patients with kidney disease and their families providing the clinical staff with information about sleep habits. Although due to the cross-sectional nature of our data no conclusions can be drawn regarding causal relationships, it was obvious from several of the situational descriptions the patients provided that they regarded their itching as being the cause of their sleep disturbances. As Murphy and Carmichael (2000) pointed out, renal itching can be a major cause of morbidity in patients with chronic renal failure. Ekelund et al. (2004) also suggested itching to be associated with decreased survival. Additional studies are needed to elucidate the aetiology of itching and the relations of itching to sleep behaviour and to physical and mental well-being generally.

Not surprisingly, uncertainty regarding the future was prevalent in the dialytic patients, the frustration associated either with renal therapy of any type or with waiting for a transplantation being a prominent issue in the descriptions the patients provided. Such uncertainty or frustration may also give rise to dependency or to avoidance coping. As Welch and Austin (2001) noted, avoidance coping may account for much of the relationship between psychosocial stressors and depression symptoms. For many of the patients, financial problems due to the diminished ability of the patients of normal working age (18-65) to undertake paid employment were salient. Also, as could be expected, the employment rate of the dialysis patients was significantly lower than that of the patients in the predialysis group. Although the percentage employed was very low in both the predialysis and the dialysis groups, this may possibly be explained in part by the inclusion in the study of patients who had reached pension age, generally 65 in Sweden but in some cases several years earlier. The system of universal health insurance and social benefits in Sweden may well have removed the worst economic consequences of having a chronic disease. However, patients may nevertheless have some difficulties regarding economic matters, as evidenced by some of the examples provided, since the insurance benefits received are based on the income received earlier. This is in line with findings in Sweden that, despite the welfare system, long- 
term illness increases the risk of adverse financial conditions, unemployment, and of not being economically active (Lindholm, Burström, \& Diedrichsen, 2001).

The finding of the duration of kidney disease for women being longer in the predialysis than the dialysis sample merits further study. No further check was made of the clinical data on the subjective duration of kidney disease. Gender differences involving women tending to seek both health care generally and health care for minor health problems more frequently than men do may be involved here. The presence of other illness, such as urinary tract infection, could possibly explain the finding of the duration of the disease being significantly longer in the predialytic women than in those with dialysis. In addition, post-hoc inspection of the comorbidity data indicate systemic disease (such as amyloidosis) to be more prevalent in predialytic women than in predialytic men, which may be the reason for the earlier detection of kidney disease in the former than in the latter group. In this connection, one should also note the recent findings of longer predialytic nephrological care, of at least several years duration, being associated with lower mortality and morbidity rates in dialysis patients (Górriz, Sancho, Pallardó, Amoedo, Barril, Salgueira, \& de la Torre, 2002).

One should note that the most frequently endorsed stressors, such as fatigue, effects on sexuality and inability to work, were not regarded as extremely stressful by the patients, the group scores on the stressfulness scale being around 50. However, the mean stressfulness scores obtained for the patients in the present study were considerably higher than those in a group of 30 cancer outpatients, predominantly with breast or head-and-neck cancer, studied by Andersson and Albertsson (2000), but were considerably lower than those reported in a study of 260 patients with fibromyalgia (Andersson, 2000), the questionnaires involved being either the same or very similar in both cases. Thus, for example, in the study of cancer outpatients the mean stressfulness score for fatigue was found to be 27.6, the corresponding value in the study 
of fibromyalgia patients being 78.9. The relatively low stressfulness values that Andersson and Albertsson (2000) found in cancer patients, both for the predominantly physical and the predominantly psychological or psychosocial types of stressors, are in line with the somewhat counterintuitive finding of patients with cancer not being significantly more distressed in subsequent phases of the disease than individuals in the general population (see Sprangers, Tempelaar, van den Heuvel \& de Haes, 2002). As pointed out by Sprangers et al. (2002), who based their studies on crisis theory, post-crisis cancer patients, especially those with a high level of self-esteem, appear to have succeeded in making their coping resources more effective than those of healthy individuals. The daily or nearly daily dependency of dialysis patients on medical technology may be particularly distressing, few if any parallels to such dependency being seen in other types of chronic illness (cf. Sensky, 1993), This may at least partly explain the mean stressfulness scores being higher in the pre-dialysis and dialysis patients than in the cancer out-patients with regard to fatigue and other symptoms prevalent in chronic disease. The finding of the mean scores being higher in the dialysis than in the pre-dialysis sample further supports the idea of ESRD not being a "typical" physical illness. The day-today dependency of the ESRD patient on a life-saving machine extends not only to the medical unit staff but also, in many cases, to family members. As Sensky (1993) has pointed out, the patient may show no signs of illness between the dialysis sessions, leading to others putting pressure on them to live a "normal" life, the strain on them this produces possibly giving rise to problems of a "double bind" or "sick role cycle" type. One should note, however, that in our study there is considerable variability in the stressfulness ratings obtained, as the high standard deviations indicate. One can speculate, in line with Devins, Beanlands, Mandin and Paul (1997), that differences among chronic kidney patients in how illness-induced life-style disruptions are understood and in their self-concept may serve to explain the marked variability referred to in connection with fatigue and other either illness- or non-illness-related factors. On the other hand, in chronic widespread pain such as fibromyalgia, illness-induced symptoms may be exaggerated due to the condition not manifesting itself in bodily symptoms (see Andersson \& Hovelius, 2005). 
Finally, one should note that the dialysis patients were generally on hemodialysis at the time of testing and that, due to the only small number of patients with peritoneal dialysis (16 altogether), the two dialysis groups were collapsed into one. Further studies are needed to explore more fully what possible differences there are between patients on hemodialysis and on peritoneal dialysis in the prevalence of symptoms, although one should note that Parfrey et al. (1988) found little difference between these two groups in the prevalence of the most important symptoms that they noted in their patients. Also, in Sweden, the guiding principles for the care of nephrology patients consider hemodialysis and peritoneal dialysis to be integrated parts of an optimal treatment of chronic renal failure (Svensk Njurmedicinsk Förening, 2000). 


\section{References}

Andersson, S. I. (1996). Appraisal, coping, motivational factors and gender in vocational rehabilitation. Scandinavian Journal of Social Medicine, 24, 161-168.

Andersson, S. I. (2000). Stress, coping and household work: The fibromyalgia example. Journal of Tokyo Medical University, 58, 377-383.

Andersson, S. I., \& Albertsson, M. (2000). Stress and situationally related coping in cancer out-patients and their spouses. Stress Medicine, 16, 209-217.

Andersson, S. I. \& Hovelius, B. (2005). Illness-related complaints in women with chronic widespread pain: Importance of a contextual approach. Stress and Health, 21, 235-244.

Andersson, S. I., Manthorpe, R., \& Theander, E. (2001). Situational patterns in coping with primary Sjögren's syndrome. Psychology, Health \& Medicine, 8, 29-40.

Brunier, G. M., \& Graydon J. (1993). The influence of physical activity on fatigue on patients with ESRD on hemodialysis. ANNA Journal, 20, 457-461.

Devins, G. M., Beanlands, H., Mandin, H., \& Paul, L. C. (1997). Psychosocial impact of illness intrusiveness moderated by self-concept and age in end-stage renal disease. Health Psychology, 16, 529-538.

Devins, G. M., Mann, J., Mandin, H., Paul, L. C., Hons, R. B., Burgess, E. D., Taub, K., Schorr, S., Letourneau, P. K., \& Buckle, S. (1990). Psychosocial predictors of survival in end-stage renal disease. Journal of Nervous and Mental Disease, 178, 127-133.

Edgell, E. T., Coons, S. J., Carter, W. B., Kallich, J. D., Mapes, D., Damush, T. M., \& Hays, R. D. (1996). A review of health-related quality-of-life measures used in end-stage renal disease. Clinical Therapeutics, 18, 887-938.

Ekelund, M. L., Westman, K., \& Andersson, S. I. (2004). Dialysis-linked complaints and burdens of illness on patient and spouse as predictors of survival in end-stage renal disease patients with home 
hemodialysis: a 10-year follow-up study. Stress and Health, 20, 29-34.

Górriz, J. L., Sancho, A., Pallardó, L. M, Amoedo, M. L., Barril, G., Salgueira, M., \& de la Torre, M. (2002). Longer pre-dialysis nephrological care is associated with improved long-term survival of dialysis patients. More facts. Nephrology Dialysis Transplantation, 17, 1354-1355.

Iliescu, E. A., Coo, H., McMurray, M. H., Meers, C.L., Quinn, M. M., Singer, M. A., \& Hopman, W. M. (2003). Quality of sleep and health-related quality of life in haemodialysis patients. Nephrology Dialysis Transplantation, 18, 126-132.

Kimmel, P. L. (2000a). Just whose quality of life is it anyway? Controversies and consistencies in measurements of quality of life. Kidney International, 57 (Supplement 74), S113-S120.

Kimmel, P. L. (2000b). Psychosocial factors in adult end-stage renal disease patients treated with hemodialysis: Correlates and outcomes. American Journal of Kidney Diseases, 35(Suppl. 1), 132-140.

Kimmel, P. L. (2001). Psychosocial factors in dialysis patients. Kidney International, 59, 1599-1613. Lindholm, C., Burström, B., \& Diedrichsen, F. (2001). Does chronic illness cause adverse social and economic consequences among Swedes? Scandinavian Journal of Public Health, 29, 63-70.

McCann, K., \& Boore, J. R. P. (2000). Fatigue in persons with renal failure who require maintenance haemodialysis. Journal of Advanced Nursing, 32, 1132-1142.

McGee, H., \& Bradley, C. (Eds). (1994). Quality of life following renal failure. Psychosocial challenges accompanying high technology medicine. Chur, Switzerland: Harwood Academic Publishers.

Murphy, M., \& Carmichael, A. J. (2000). Renal itch. Clinical \& Experimental Dermatology, 25, 103-106.

Palmer, B. F. (1999). Sexual dysfunction in uremia. Journal of the American Society of Nephrology, 10, $1381-1388$

Parker, K. P. (1997). Sleep and dialysis: a research-based review of the literature. ANNA Journal, 24, 
626-641.

Parfrey, P. S., Vavasour, H. M., Henry, S., Bullock, M, \& Gault, M. H. (1988), Clinical features and severity of non-specific symptoms in dialysis patients. Nephron, 50, 121-128.

Sensky, T. (1993). Psychosomatic aspects of end-stage renal failure. Psychotherapy and Psychosomatics, 59, 56-68.

Sprangers, M. A. G., Tempelaar, R., van den Heuvel, W. J. A., \& de Haes, H. C. J. M. (2002). Explaining quality of life with crisis theory. Psycho-Oncology, 11, 419-426.

SRAU (Svenskt register för aktiv uremivård). (2002). Aktiv uremivård i Sverige 1991-2001. Skövde, Sweden: Author.

Svensk Njurmedicinsk förening. (2000). Riktlinjer för omhändertagande av patienter med njursvikt. Stockholm: Author.

Unruh, M. L., Weisbord, S. D., \& Kimmel, P. L. (2005). Health-related quality of life in nephrology research and clinical practice. Seminars in Dialysis, 18, 82-90.

Weisbord, S. D., Fried, L. F., Arnold, R. M., Rotondi, A. J., Fine, M. J., Levenson, D. J., \& Switzer, G. E. (2004). Development of a symptom assessment instrument for chronic hemodialysis patients: The Dialysis Symptom Index. Journal of Pain and Symptom Management, 27, 226-240.

Welch, J. L, \& Austin J. K. (2001). Stressors, coping and depression on haemodialysis patients. Journal of Advanced Nursing, 33, 200-207.

Wiberg, E. M. (2003). Viktigt att finna bra träningsformer för dialyspatienter (Well-designed training programs crucial for dialysis patients). Läkartidningen, 100, 519-526.

Williams, S. W., Tell, G. S., Zheng, B , Shumaker, S., Rocco, M. V., \& Sevick, M. A. (2002). Correlates of sleep behavior among hemodialysis patients. The Kidney Outcomes Prediction and Evaluation (KOPE) Study. American Journal of Nephrology, 22, 18-28. 
Table 1. Demographic and clinical characteristics of the predialysis and dialysis samples

\begin{tabular}{llll}
\hline & & \\
Variable & $(n=72 ;$ & Dialysis patients & P-value \\
& 55 men, 17 women $)$ & 51 men; 22 women $)$ & \\
\hline
\end{tabular}

\section{Sociodemographic}

Age, years mean (SD)

Men

$60.2(13.18)$

$58.6(13.49)$

NS

Women

$50.6(17.37)$

$55.9(15.54)$

NS

Education, years mean (SD)

Men

$9.6(3.15)$

$9.2(2.72)$

NS

Women

$9.8(2.51)$

$\%$ Living alone

25.0

$8.7(2.03)$

30.0

NS

$\%$ Employed

27.9

13.6

NS

$\%$ Manual workers

52.8

46.4

$<0.05$

NS

\section{Clinical}

Duration of kidney

disease, years mean (SD)

Men

$11.4(12.76)$

$10.2(10.97)$

NS

Women

$14.2(7.72)$

8.7 (9.83)

$<0.05$

$\%$ Comorbidity $^{\mathrm{a}}$

43.1

52.0

NS

\footnotetext{
${ }^{a}$ A measure based on the presence of other chronic disease (mainly diabetes mellitus, rheumatoid arthritis, or chronic obstructive pulmonary disease). For the predialysis and the dialysis group of diabetes mellitus patients, $n=18$ ( 15 men, 3 women) and 17 ( 10 men, 7 women), respectively. For cardiovascular disease there were seven men and one woman in the predialysis group and eight men and five women in the dialysis group. For cancer there were no men but three women (two of whom had uterus cancer) in the predialysis group and three men (one with colon cancer, one with non-Hodgkins lymphoma and one with prostate cancer) and one woman (with bladder cancer) in the dialysis group. For rheumatoid arthritis there was one man (no women) in the predialysis group and two men (no women) in the dialysis group.
} 
Table 2. Frequency (\%) and mean stressfulness scores (0-100) of stressors as reported by pre-dialytic (Pre-DP) $)^{\mathrm{a}}$ and dialytic (DP) $)^{\mathrm{b}}$ patients, and rotated factor loadings of the stressfulness scores

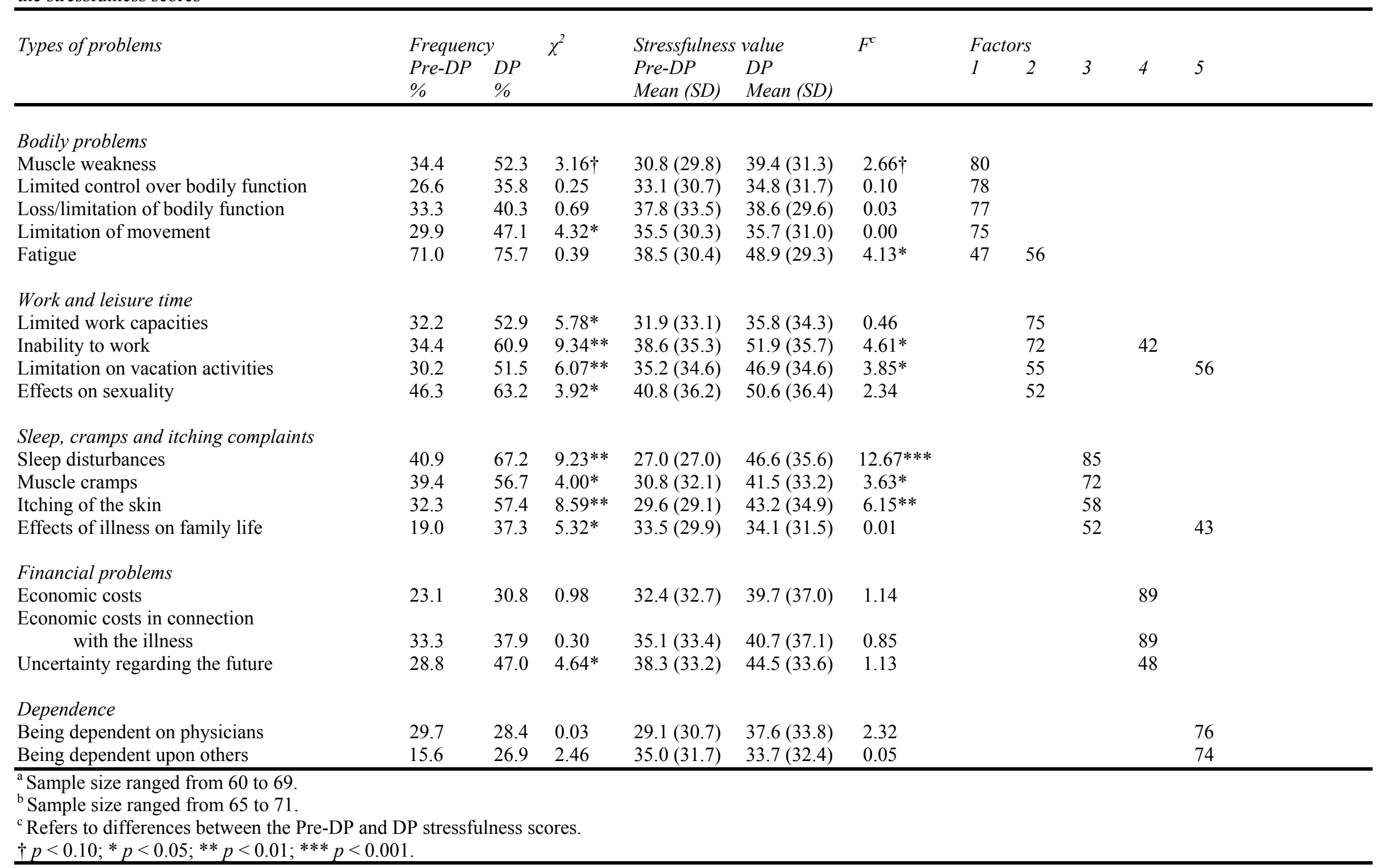

